\title{
Severe neonatal-onset encephalopathy with microcephaly
}

INSERM

\section{Source}

INSERM. (1999). Orphanet: an online rare disease and orphan drug data base. Severe neonatal-onset encephalopathy with microcephaly. ORPHA:209370

Severe neonatal-onset encephalopathy with microcephaly is a rare monogenic disease with epilepsy characterized by neonatal-onset encephalopathy, microcephaly, severe developmental delay or absent development, breathing abnormalities (including central hypoventilation and/or respiratory insufficiency), intractable seizures, abnormal muscle tone and involuntary movements. Early death is usual. 